\title{
Supernumerary Cerebellar Vermis: A Unique Cerebellar Anomaly
}

\author{
Tahani Ahmad, Charles Raybaud, Daniel McNeely, Naeem Khan, Mustafa Nadi
}

Keywords: Cerebellum, Anomaly, Vermis, Development, Molecular boundaries, Signaling effect

doi:10.1017/cjn.2019.2

Can J Neurol Sci. 2019; 46: 255-257

\section{Case Presentation}

An 11-year-old female patient presented to our center for ventriculoperitoneal shunt check-up after a period of unexplained nausea. The patient's family immigrated from another country 1 year before the presentation. The patient received the first shunt at the age of 1 week for aqueductal stenosis that was revised after 6 months for a shunt infection. She experienced an uneventful period until this presentation. At our facility, shunt series (X-ray) and brain MRI were performed, and shunt failure was excluded. Interestingly, her brain MRI revealed a unique cerebellar anomaly that was not reported in her previous medical documents. The patient's developmental assessment revealed that she has learning difficulties.

\section{Neuroimaging: Dr. Ahmad}

The MRI showed an extra-vermis attached to the anterior lobe of the native vermis, mainly to the culmen (Figure 1A,B). The anterior superior folia of this extra-cerebellar tissue herniate superiorly through the left side of the tentorial notch (Figure 1D,E). The extra-vermis has a primary fissure and a pre-pyramidal fissure. It demonstrates a similar signal intensity to the native one on all sequences (Figure 1C,F and G). Additionally, there is an aqueductal stenosis and corpus callosum dysgenesis (Figure 1C). ${ }^{1}$

\section{Discussion: Dr. RaYbaud and Dr. Ahmad}

The extra-vermis is believed to be the result of a developmental error that occurs at the early stages of embryogenesis. ${ }^{2}$ For better understanding of this anomaly, the morphological and molecular aspects of cerebellar development are discussed here.

\section{Morphological Aspect}

The mesencephalon (midbrain (MB)) and metencephalon (upper hindbrain (HB)) contribute to form the cerebellum during the fifth week of gestation. The alar plates of the mesencephalon contribute to cerebellar vermis. ${ }^{3}$ The rhombic lips are the primordia of the cerebellar hemispheres and the source of granule cells ${ }^{4}$.
The rhombomeres 1-11 (r1-r11) result from the primary segmentation of the brainstem, initiated by the isthmic organizer. Rhombomeres are identified as $\mathrm{r} 0$ (the isthmus: the junction between $\mathrm{MB}$ and $\mathrm{HB}$ ) followed by $\mathrm{r} 1-\mathrm{r} 11$. The cerebellum originates from $\mathrm{r} 0-\mathrm{r} 1$. It is generally considered that the vermis originates from the alar plate of $\mathrm{r} 0$ and the hemispheres from the alar plate of $\mathrm{r}^{3} .^{3}$

The primary fissure is the first to develop at 14 weeks. The pre-pyramidal and the secondary (post-pyramidal) fissures are seen at 16 weeks (Figure 2). The posterolateral fissure is visible at week $17 .^{2}$ The earliest studies of gestational age demonstrate that the ratio between the volume of the parts above and the volume of those below the primary fissure is 1:1 until the age of 16 weeks. This will be followed by rapid growth of the pyramid vermis and tuber vermis. The parts beneath the primary fissure grow faster and become larger than the parts above the fissure. Figure 3 illustrates the normal anatomy of the vermis.

\section{Molecular Aspect: Dr. RaYbaud and Dr. Nadi}

Three phases can be recognized:

I. Allocation of the cerebellar anlage (CA): The CA is a product of the early embryonic segmental phase that results in rhombomeric subdivisions within the HB just after neural tube closure. The territory that generates the $\mathrm{CA}$ is close to the boundary (the isthmus) between the $\mathrm{MB}$ and the HB. The patterning in this phase is significant for the vermis evolution. ${ }^{5}$

II. The process of cell type allocation: Generation of neuronal diversity along the dorsoventral axis. ${ }^{6}$

III. The transit amplification process. It extends up to two years postnatally in humans. ${ }^{5}$ We will elaborate further on phase I because the anomaly in this case is mostly due to an event that happened in the early weeks.

The CA sits between Otx and Hox domains, a region that lacks expression of Otx and Hox genes. The majority of the cerebellum arises from the rostral metencephalon and one-third of cerebellar granule cells originate from the mesencephalon. ${ }^{7}$ More precisely, it arises between the $\mathrm{MB}$ and $\mathrm{HB}^{8}{ }^{8}$ There are certain genes that

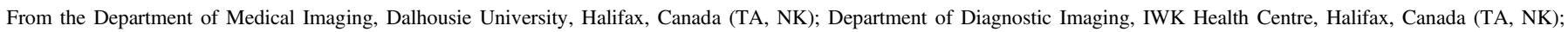

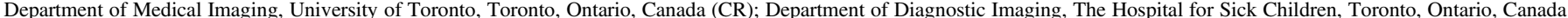

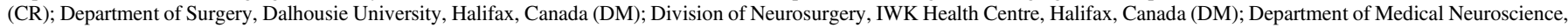
Dalhousie University, Halifax, Canada (MN); Division of Neurosurgery, QE II Health Sciences Centre, Halifax, Canada (MN).

Received July 17, 2018. Final Revisions Submitted December 22, 2018. Date of Acceptance January 9, 2019.

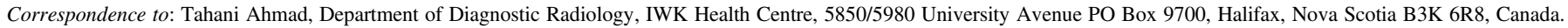
Email: tahani.ahmad@iwk.nshealth.ca 

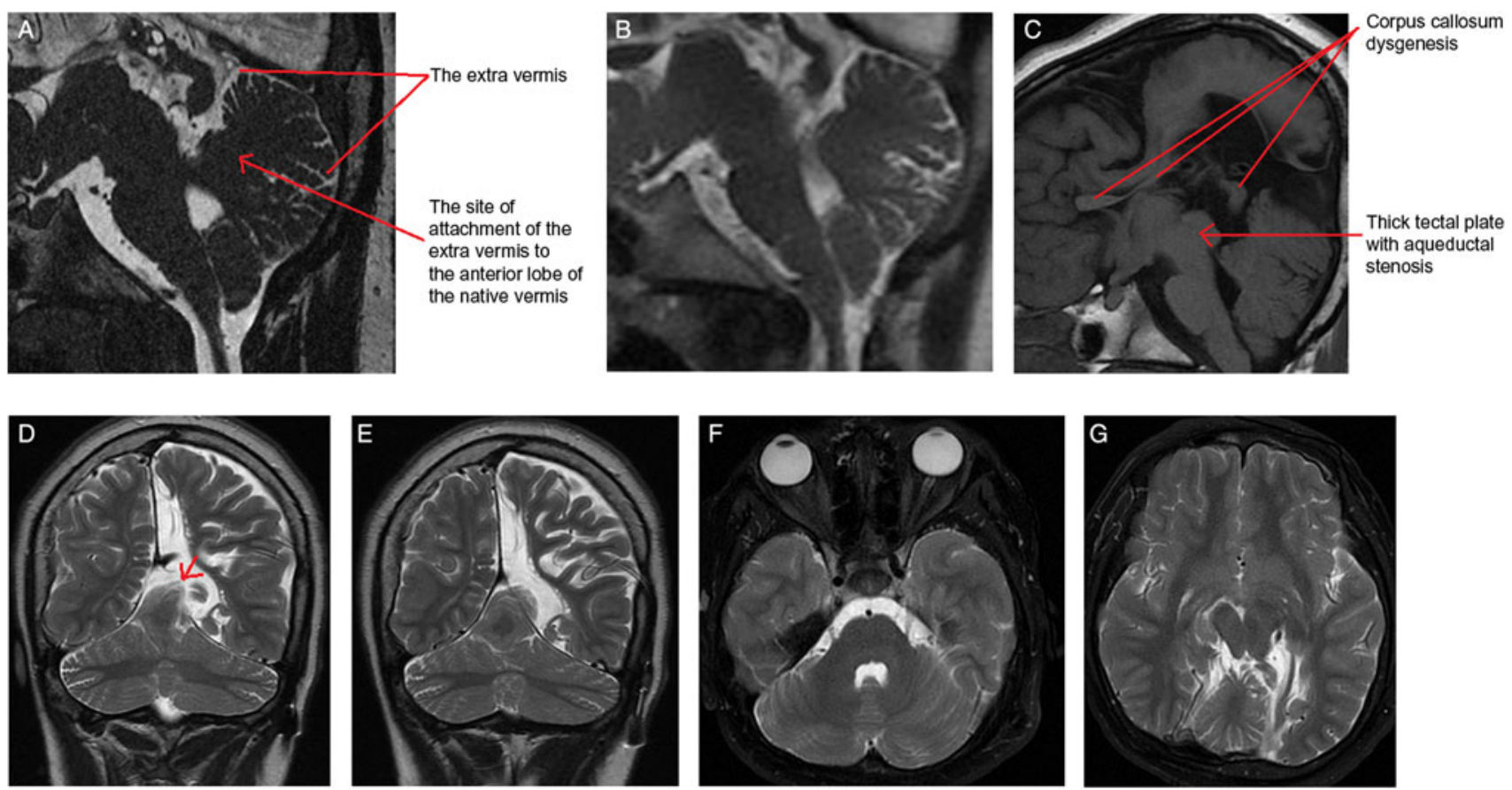

Figure 1: (A) Sagittal FIESTA image - slightly off midline - through the cerebellum demonstrates the extra vermis that is attached to the culmen of the native vermis. Notice the unique finding that the supernumerary vermis is developed from the dorsal aspect of the native vermis with a very clear site of attachment to the culmen and not from the brain stem. (B) Sagittal midline T2-weighted image shows the cerebellar vermian anomaly. (C) Sagittal T1-weighted MR image re-demonstrates the same findings, and shows the corpus callosum dysgenesis and the thick tectal plate with the resultant aqueductal stenosis. The anomalous vermis exhibits a similar signal intensity to the native one on coronal T2-weighted images ( $D$ and E). Coronal T2 weighted MRI images show the anomalous vermis herniated through the tentorium (red arrow in D). (F and $G$ ) Axial T2-weighted images show the normal morphology of the cerebellar hemispheres and re-demonstrate the extra vermis.

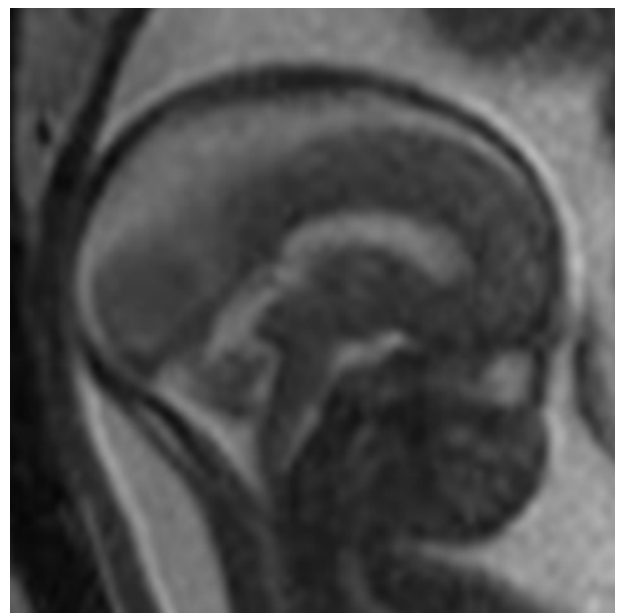

Figure 2: Midline sagittal FIESTA MRI image through the vermis in a 23-week fetus demonstrating the development of the major fissures and the vermian segments by this gestational age.

determine the cerebellar territories: Otx2 at the rostral boundary, Hoxa2 at the caudal boundary, and Gbx2 which is expressed in $\mathrm{r} 1$, abutting Otx2. Otx2 is required to establish forebrain and $\mathrm{MB}$ territories, and its absence causes a rostral expansion of the cerebellar Gbx2-positive territory at the expense of MB tissue. Contrariwise, ectopic expression of Otx2 in the rostral HB transforms this region into a Gbx2-negative $\mathrm{MB}$ with a caudal shift in the position of the MB-HB boundary (MHB). ${ }^{9}$ Thus, it is an essential requirement that Otx2 is absent for cerebellar differentiation to begin. On the other hand, while $\mathrm{Gbx} 2$ is required for

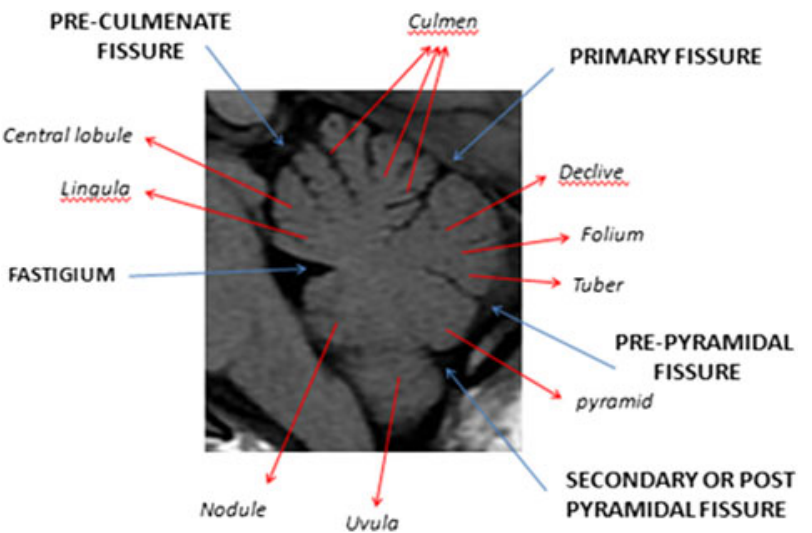

Figure 3: Annotated sagittal T1 MRI image of the cerebellum in a healthy teenage volunteer showing normal anatomy and major fissures.

cerebellar formation, ${ }^{10}$ Hoxa2 determines the caudal limits of the cerebellum. ${ }^{11}$

Additionally, the morphogen fibroblast growth factor 8 (Fgf8) and $\mathrm{Gbx} 2^{12}$ promote cell sorting and lineage restriction at the boundary. Fgf8 signaling has a significant role in allocating isthmic territory and the origins of the vermis in the MHB. Fgf8 is expressed within the Gbx2-positive domain and essential for the survival of the isthmic region. ${ }^{13}$ It maintains $\mathrm{r} 1$ as an Otx2negative domain. ${ }^{14}$ The ectopic Fgf8 in the MB can induce a secondary cerebellum, whereas loss of Fgf8 expression induces vermis dysplasia ${ }^{13}$ and expansion of the Otx2 domain. ${ }^{15}$ Sprout genes are Fgf8 antagonists and regulate the levels of fibroblast 
growth factor signals from the $\mathrm{r} 0 .^{5}$ Mutations in sprout 2 genes (Spry2) result in expansion of the vermis. ${ }^{16}$

Interestingly, the extra vermis in our case arises from the dorsal aspect of the native vermis and not from the brain stem as it would be expected from the molecular development explained above in the cases of overexpression of Gbx2. This is likely attributed to a mutation in either Fgf8 or spry 2 genes that alter the allocation of the isthmus and the origin of the extra vermis. This extra vermis showed a well-formed culmen and a central lobule as well as a primary fissure, and a pre-pyramidal fissure with no formation of the posterior lobe. This suggests that the anomaly has likely developed before the 16 weeks of gestation.

\section{Review of Literature: Clinical Implications and Genetic Association}

While no similar case of extra vermis has been described in the literature, there are few reported cases of extra cerebellar hemispheres. In 1990, Jackson et al. reported a case of duplication of a unilateral cerebellar hemisphere associated with a complex ipsilateral ear anomaly. ${ }^{17}$ The author traced the timing of the problem to the third week of gestation. Clinically, patient's examination was significant to lower extremities hyper-reflexia, truncal hypotonia wide-base gait with right upper limb intentional tremors. Moreover, the patient's IQ was low-average and he was delayed in terms of fine and gross motor skills as well as social adaptation. Another case report published in 2011 described a 16-month-old girl with a third cerebellar hemisphere and incompletely formed superior vermis. ${ }^{18}$ This was attributed to a mutation in genes expressed during early cerebellar development leading to an extra RL growth. The anomaly impacted the patient's motor milestone as she was unable to stand. This child also showed mild truncal ataxia, generalized hypotonia, and diminished deep tendon reflexes. Furthermore, Hattapoğlu et al. reported a third case of supernumerary hemi-cerebellum in an adult female patient who presented with headaches and episodes of syncope. ${ }^{3}$ Neurological exam was intact apart from a slight increase in the deep tendon reflexes. It is noteworthy that none of these three cases or our patient had language skills issues.

Several cerebellar anomalies can be associated with other congenital anomalies and there are different genetic defects responsible for them, ${ }^{19}$ and further elaboration of various types of malformations and their genetic bases is out of the scope of this quick highlight. However, in regard to the discussed three reported cases of extracerebellar tissue, no specific genetic alterations or dedicated genetic testing was reported. ${ }^{3,17,18}$ Jackson et al examined their patients and showed normal male chromosomes $46 \mathrm{XY} .{ }^{17}$

\section{Conclusion}

The purpose of this case review is to share an unusual cerebellar malformation with the medical community and to shed light on the potential embryological errors that could result in this anomaly. It is expected that more cases will be reported in the future with further advancement of diagnostic imaging techniques.

\section{Authors' Declarations}

All authors declare no conflict of interest in this work. There was no financial support or grant for this production. This work has never been presented before in part or whole.

\section{Ethical Standards Statement}

No patient's identification information was used. A consent form from the patient's guardian was obtained.

\section{REFERENCES}

1. Hanna RM, Marsh SE, Swistun D, et al. Distinguishing 3 classes of corpus callosal abnormalities in consanguineous families. Neurology. 2011;76(4):373-82.

2. Liu F, Zhang Z, Lin $X$, et al. Development of the human fetal cerebellum in the second trimester: a post mortem magnetic resonance imaging evaluation. J Anat. 2011;219(5): 582-8.

3. Hattapoglu S, Hamidi C, Goya C, Cetincakmak MG, Teke M, Ekici F. A surprising case: a supernumerary heterotopic hemicerebellum. Clin Neuroradiol. 2015;25(4):431-4.

4. Dun XP. Origin of climbing fiber neurons and the definition of rhombic lip. Int J Dev Neurosci: Off J Int Soc Dev Neurosci. 2012;30(5):391-5.

5. Butts T, Green MJ, Wingate RJ. Development of the cerebellum: simple steps to make a 'little brain'. Development. 2014;141(21): 4031-41.

6. Simon H, Hornbruch A, Lumsden A. Independent assignment of antero-posterior and dorso-ventral positional values in the developing chick hindbrain. Curr Biol. 1995;5(2):205-14.

7. Hallonet ME, Teillet MA, Le Douarin NM. A new approach to the development of the cerebellum provided by the quail-chick marker system. Development. 1990;108(1):19-31.

8. Millet S, Bloch-Gallego E, Simeone A, Alvarado-Mallart RM. The caudal limit of Otx2 gene expression as a marker of the midbrain/hindbrain boundary: a study using in situ hybridisation and chick/quail homotopic grafts. Development. 1996;122(12): 3785-97.

9. Katahira T, Sato T, Sugiyama S, et al. Interaction between Otx 2 and Gbx2 defines the organizing center for the optic tectum. Mech Dev. 2000;91(1-2):43-52.

10. Wassarman KM, Lewandoski M, Campbell K, et al. Specification of the anterior hindbrain and establishment of a normal mid/ hindbrain organizer is dependent on Gbx2 gene function. Development. 1997;124(15):2923-34.

11. Gavalas A, Davenne M, Lumsden A, Chambon P, Rijli FM. Role of Hoxa-2 in axon pathfinding and rostral hindbrain patterning. Development. 1997;124(19):3693-702.

12. Tossell K, Kiecker C, Wizenmann A, Lang E, Irving C. Notch signalling stabilises boundary formation at the midbrainhindbrain organiser. Development. 2011;138(17):3745-57.

13. Basson MA, Echevarria D, Ahn CP, et al. Specific regions within the embryonic midbrain and cerebellum require different levels of FGF signaling during development. Development. 2008;135(5): 889-98.

14. Foucher I, Mione M, Simeone A, Acampora D, Bally-Cuif L, Houart C. Differentiation of cerebellar cell identities in absence of Fgf signalling in zebrafish Otx morphants. Development. 2006; 133(10): 1891-900.

15. Sato T, Joyner AL. The duration of Fgf8 isthmic organizer expression is key to patterning different tectal-isthmo-cerebellum structures. Development. 2009;136(21):3617-26.

16. Yu T, Yaguchi Y, Echevarria D, Martinez S, Basson MA. Sprouty genes prevent excessive FGF signalling in multiple cell types throughout development of the cerebellum. Development. 2011; 138(14):2957-68.

17. Jackson JM, Sadove AM, Weaver DD, Edwards MK, Bull MJ. Unilateral duplication of the cerebellar hemisphere and internal, middle, and external ear: a clinical case study. Plast Reconstr Surg. 1990;86(3):550-3.

18. Agarwal S, Gathwala G. Third cerebellar hemisphere: an unusual new cerebellar anomaly. AJNR Am J Neuroradiol. 2011;32(4): E73-4.

19. Aldinger KA, Doherty D. The genetics of cerebellar malformations. Semin Fetal Neonatal Med. 2016;21(5):321-32. 